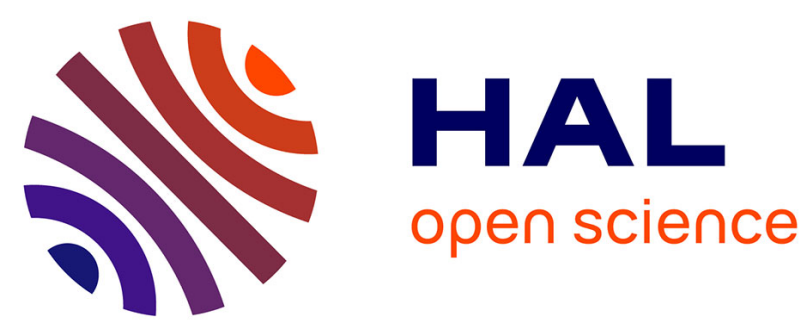

\title{
Analysis of the modal content into large-mode-area photonic crystal fibers under heat load
}

Enrico Coscelli, Romain Dauliat, Federica Poli, Dia Darwich, Annamaria

Cucinotta, Stefano Selleri, Kay Schuster, Aurélien Benoit, Raphaël Jamier, Philippe Roy, et al.

\section{To cite this version:}

Enrico Coscelli, Romain Dauliat, Federica Poli, Dia Darwich, Annamaria Cucinotta, et al.. Analysis of the modal content into large-mode-area photonic crystal fibers under heat load. IEEE Journal of Selected Topics in Quantum Electronics, 2015, 22 (2), 10.1109/JSTQE.2015.2479156 . hal-01239449

\section{HAL Id: hal-01239449 \\ https://hal.science/hal-01239449}

Submitted on 7 Dec 2015

HAL is a multi-disciplinary open access archive for the deposit and dissemination of scientific research documents, whether they are published or not. The documents may come from teaching and research institutions in France or abroad, or from public or private research centers.
L'archive ouverte pluridisciplinaire $\mathbf{H A L}$, est destinée au dépôt et à la diffusion de documents scientifiques de niveau recherche, publiés ou non, émanant des établissements d'enseignement et de recherche français ou étrangers, des laboratoires publics ou privés. 


\title{
Analysis of the modal content into large-mode-area photonic crystal fibers under heat load
}

\author{
Enrico Coscelli, Member, IEEE, Romain Dauliat, Federica Poli, Dia Darwich, \\ Annamaria Cucinotta, Member, IEEE, Stefano Selleri, Senior Member, IEEE, Kay Schuster, \\ Aurélien Benoît, Raphael Jamier, Philippe Roy, François Salin
}

\begin{abstract}
Thanks to their capability to provide very large mode area together with effective suppression of high-order modes, while allowing strong pump absorption and efficient conversion, Yb-doped double-cladding photonic crystal fibers are one of the key enabling factors for the development of high power fiber lasers. Thermal effects are currently appointed as the main bottleneck for future power scaling since, beyond a certain average power, they allow guidance of high order modes and energy transfer to them, causing a sudden degradation of the beam quality. In this paper the effects of heat load on the modes of double cladding fibers are thoroughly analyzed with a full-vector modal solver based on the finite-element method with integrated steady-state heat equation solver. Fibers with different inner cladding designs are compared to provide a deeper understanding of the mechanisms beyond the mode reconfinement and coupling. The influence of the fiber design on the robustness of the single-mode regime with respect to fiber heating has been demonstrated, providing a clear picture of the complex interaction between modes. On the basis of simulation results it has been possible to group fiber modes into three families characterized by peculiar reaction to heating.
\end{abstract}

Index Terms-Photonic crystal fibers, thermo-optic effect, fiber lasers and amplifiers.

\section{INTRODUCTION}

$\mathbf{T}$ HE last decade has witnessed a huge progress of the performances of high power fiber lasers and amplifiers [1][3]. The output power of CW near-diffraction limited fiber lasers has been increasing by about $2 \mathrm{~dB} /$ year over the last two decades, and it is currently exceeding $20 \mathrm{~kW}$ in commercial systems [4]. Substantial improvements have been achieved also in the pulsed regime. Q-switched and master oscillator-power amplifier have demonstrated the capability to emit pulses with duration shorter than $10 \mathrm{~ns}$ and energy exceeding $33 \mathrm{~mJ}$ [5], as well as $60 \mathrm{~ns}$ long, $26 \mathrm{~mJ}$ pulses with average power of $130 \mathrm{~W}$ [6]. In the ultrashort pulse regime, the introduction of chirped pulse amplification technique has paved the way to the generation of sub-500 fs pulses with $\mathrm{mJ}$ energy and $22 \mathrm{GW}$ peak power [7]. Such performances have been made possible by constant innovations in the Yb-doped fiber technology.

E. Coscelli, F. Poli, A. Cucinotta and S. Selleri are with the Information Engineering Department, University of Parma, I-43124 Parma, Italy (e-mail: enrico.coscelli@unipr.it).

R. Dauliat, Dia Darwich, Aurelién Benoît, Raphael Jamier and P. Roy are with Xlim Research Institute, Univ.Limoges, CNRS, XLIM, UMR 7252, F87000 Limoges, France.

R. Dauliat and K. Schuster are with the Leibniz Institute of Photonic Technology, D-07745 Jena, Germany.

F. Salin is with EOLITE Systems, F-33600 Pessac, France.
In particular, Double-Cladding Photonic Crystal Fibers (DCPCFs) have been one of the key enabling factors for this improvement, being able to tackle the fundamental challenge of providing a sufficient amount of pump power combined with large effective area for the signal to avoid the onset of nonlinear effects while maintaining robust Single-Mode (SM) operation, which is required to preserve laser beam quality [8], [9].

Recently, many independent works have reported severe beam pointing drifts and output power fluctuations suddenly occurring in fiber lasers operating beyond a certain average power threshold [8], [10], [11]. This phenomenon, usually referred as Transverse Mode Instability (TMI), is caused by a power transfer from the Fundamental Mode (FM) to some HighOrder Modes (HOMs), mediated by thermo-optic effect. Due to the quantum defect, a significant amount of heat is generated in the fiber core as a side effect of the optical amplification, which leads to relevant thermal lensing. As a consequence, the originally single-mode waveguide will support the propagation of HOMs, which will eventually drain power from the FM through the stimulated thermal Rayleigh scattering effect [12], [13].

One effective way to push the TMI threshold to higher power levels is to ensure that the active fiber provides strong delocalization of the HOMs even under severe heat load, while preserving high pump absorption and large core area. Several DC-PCF designs have been developed with this aim, and remarkable progress has been achieved so far exploiting the possibility of cladding microstructuration offered by stack \& draw fabrication technique [14]-[17]. Nevertheless, no thorough comparative investigation on the impact of the cladding structure on the modal content in presence of heat load due to amplification has been presented yet.

In this paper a full-vector modal solver based on the finiteelement method with embedded thermal model has been applied to three DC-PCFs, a Large-Pitch Fiber (LPF) [18], a Symmetry-Free PCF (SF-PCF) [17] and a Fully-Aperiodic LPF (FA-LPF) [19] which pursue HOM delocalization through different cladding microstructuration strategies. The effects on the guided modes of the different cladding types are investigated and compared with those obtained with a purely Thermally-Guiding Fiber (TGF) to demonstrate the combined effect of the microstructured cladding and thermally-induced refractive index change on the mode guidance.

The paper is organized as follows. The next Section will describe the considered fibers and the simulation procedure. 
Section III will discuss the modal properties of a purely thermally-guiding fiber, which will be used as a reference. In Section IV the effects of the heat load on the modal properties of DC-PCFs will be analyzed and compared. Final Section will draw the conclusion.

\section{Modeling OF LMA PCFs}

The cross-sections of the fibers that will be investigated throughout the paper, namely a Thermally-Guiding Fiber, a Large-Pitch Fiber, a Symmetry-Free PCF and a FullyAperiodic LPF, are shown in Fig. 1(a)-(d). All of them are double-cladding Yb-doped rod-type fibers, with the same doped area of $3700 \mu \mathrm{m}^{2}$, an air-cladding of $d_{A C}=255 \mu \mathrm{m}$ in diameter and a thickness of $7 \mu \mathrm{m}$. This choice ensures that in a practical laser all of them will have the same pump absorption and heat dissipation properties, since it has been demonstrated that the air-holes of the microstructured cladding does not have any significant impact on the thermal dissipation [14], [20]. Notice that rod-type fibers have a typical outer diameter of a few $\mathrm{mm}$ and are inherently stiff waveguides. As a consequence, the effects of bending will not be considered. The TGF has no inner cladding structure, and the whole cross-section has the same refractive index $n=1.45$ of undoped silica. The doped region, shown in yellow in Fig. 1 a), has a circular shape with radius $34.3 \mu \mathrm{m}$. Since there is no index contrast between the core and the inner cladding, confinement of the modes will be provided only by thermal lensing. All the other considered fibers are built over a triangular lattice stack with pitch $\Lambda=15 \mu \mathrm{m}$, where the 19 innermost cells have been replaced by index-matched Yb-doped rods. The microstructuration of the LPF, shown in Fig. 1 (b) is created by adding one air-hole with diameter $d$ every 3 cladding elements, resulting in a uniform hole-to-hole spacing $\Lambda_{L P F}=45 \mu \mathrm{m}$ with $d / \Lambda_{L P F}=0.3$, which is within the typical range of manufactured LPFs [21]. As shown in Fig. 1 (c), the air-holes of the SF-PCFs, having $d / \Lambda=0.3$, are not uniformly distributed, but have been carefully chosen to break the $C_{6 \nu}$ symmetry of the PCF lattice, which is reduced to a $C_{3}$ one [22]. Notice that the air-holes of the SF-PCF are smaller than those of the LPF, even if they are more numerous, yielding to a lower air filling fraction. Lastly, the FA-LPF, shown in Fig. 1(d), has an all-solid cladding with a raisedindex pedestal ( $n=1.457)$ matched with the gain medium and pure silica inclusions, carefully placed to avoid any mirror or rotational symmetry, to replace the air-holes.

The guided modes of the fibers have been calculated, for a wavelength of $1032 \mathrm{~nm}$, with a commercial full-vector modal solver based on the finite-element method [23], which was also used to solve the steady-state heat equation to obtain the thermally-induced refractive index change over the crosssection [20], [24]. The implemented model operates in two steps. First the temperature over the entire fiber cross-section is obtained by solving the steady-state heat equation for a given fiber geometry and heat load, assumed uniform over the doped core. Then the refractive index is pointwise updated according to the thermo-optic relation and the fiber modes are calculated. Due to the quantum-defect heating of the core
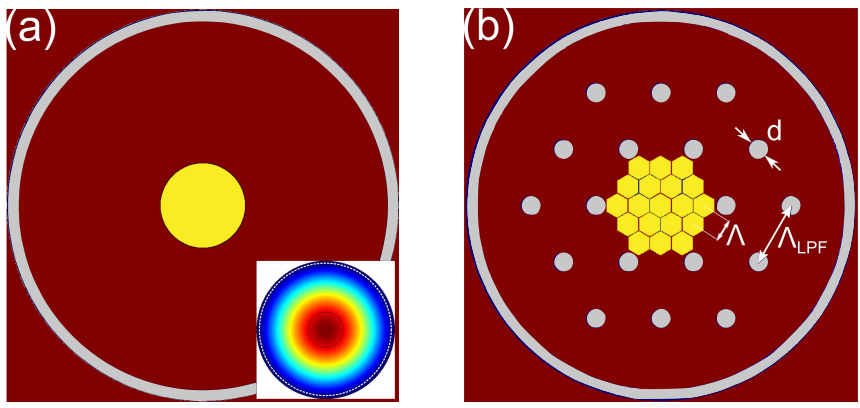

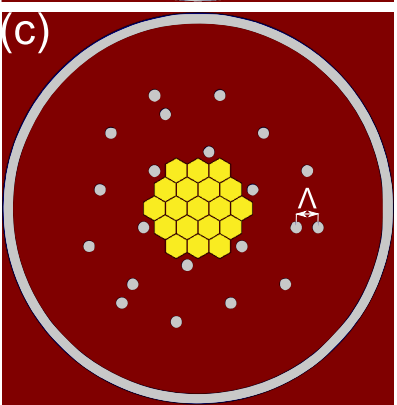

Silica glass $(n=1.45)$

Yb-doped silica $(n=1.45)$ Air

Fig. 1. Cross-section of the considered fibers: (a) Thermally-Guiding Fiber (inset: FM electric field distribution at $q=0 \mathrm{~W} / \mathrm{m}$ ); (b) Large-Pitch Fiber; (c) Symmetry-Free PCF; (d) Fully-Aperiodic LPF.

and the convective heat dissipation at the outer fiber edge, a temperature gradient is generated along the fiber crosssection. This is responsible, through the thermo-optic effect in silica, of the creation of a refractive index profile with maximum in the core center, parabolic shape in the core and logarithmic decay in the cladding [25]. This graded-index profile causes an increase of the original fiber index contrast, favoring the propagation of HOMs. The magnitude of the thermally-induced index change is proportional to the heat load $q$ generated by $\mathrm{Yb}$ ions. Assuming a pump power $P$ [W], fiber pump absorption $\alpha[\mathrm{dB} / \mathrm{m}]$, pump and laser wavelengths $\lambda_{p}$ and $\lambda_{s}$, the average heat load generated over a fiber length $d L$ is [26]:

$$
q=\frac{1-10^{-\alpha d L / 10}}{d L}\left(1-\frac{\lambda_{p}}{\lambda_{s}}\right) P
$$

The complete list of parameters used in the thermal model is reported in Table \. Notice that, with given parameters, an average heat load $q=100 \mathrm{~W} / \mathrm{m}$ over the entire fiber length corresponds to an absorbed pump power of $1390 \mathrm{~W}$. For such heat load, the maximum temperature achieved at the central point of the fiber cross section is about $311^{\circ} \mathrm{C}$, which yields a refractive index increase of about $3.3 \cdot 10^{-3}$, while it is about $21^{\circ} \mathrm{C}$ lower at the air-cladding edge. As a consequence, a refractive index gradient of about $2.4 \cdot 10^{-4}$ is created along the cross-section.

\section{THERMALLY-GUIDING FIBER}

To separate the effect on the modes of the thermallyinduced index gradient from the contribution of the cladding 
TABLE I

LIST OF THE PARAMETERS USED IN THE THERMAL MODEL

\begin{tabular}{|c|c|c|}
\hline Heat load & $q$ & $0-100 \mathrm{~W} / \mathrm{m}$ \\
Glass thermal conductivity & $k_{S i O 2}$ & $1.38 \mathrm{~W} /(\mathrm{m} \cdot \mathrm{K})$ \\
Air-clad equivalent conductivity & $k_{e q}$ & $0.085 \mathrm{~W} /(\mathrm{m} \cdot \mathrm{K})$ \\
Glass thermo-optic coefficient & $\beta$ & $1.16 \cdot 10^{-5} \mathrm{~K}-1$ \\
Fiber outer diameter & $d_{e x t}$ & $1.7 \mathrm{~mm}$ \\
Convective heat transfer coefficient & $h$ & $80 \mathrm{~W} /\left(\mathrm{m}^{2} \cdot \mathrm{K}\right)$ \\
Pump absorption & $\alpha$ & $32 \mathrm{~dB} / \mathrm{m}$ \\
Pump wavelength & $\lambda_{p}$ & $976 \mathrm{~nm}$ \\
Laser wavelength & $\lambda_{s}$ & $1032 \mathrm{~nm}$ \\
Fiber length & $d L$ & $75 \mathrm{~cm}$ \\
\hline \multicolumn{2}{|r}{} \\
\hline
\end{tabular}

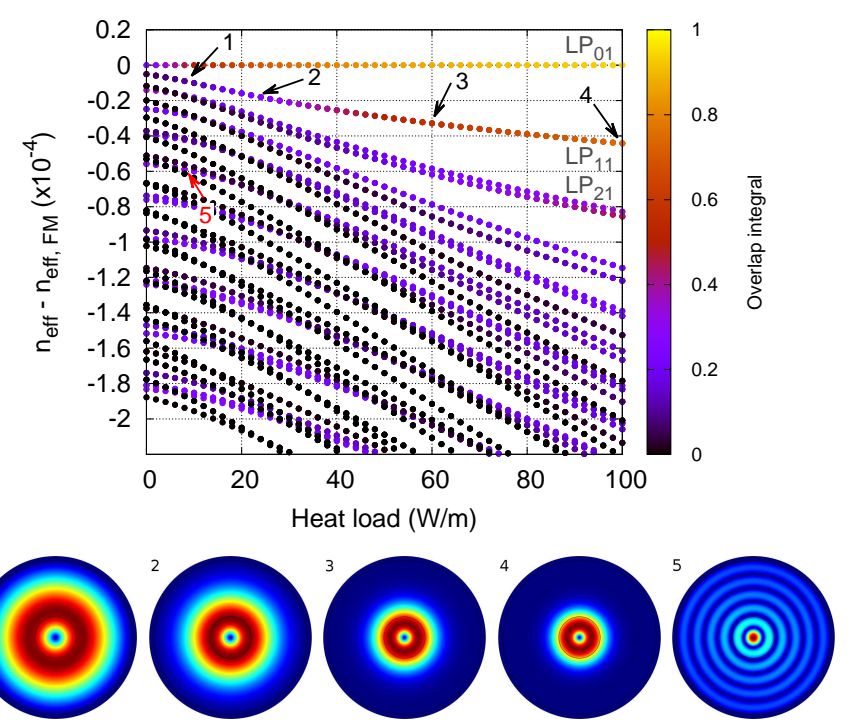

Fig. 2. Map of the modes of the thermally-guiding fiber at $1032 \mathrm{~nm}$ and sample of the electric field modulus distribution within the air cladding of the most relevant modes.

microstructuration, the TGF has been considered first. The first 150 electromagnetic modes of the TGF have been calculated over a range of heat load values spanning from $q=0 \mathrm{~W} / \mathrm{m}$ to $q=100 \mathrm{~W} / \mathrm{m}$. In absence of heat load, the fiber core and inner cladding have a uniform index, and all the solutions are confined only by the air-cladding. Nevertheless, even at $q=0 \mathrm{~W} / \mathrm{m}$ it is possible to recognize the fundamental $\mathrm{LP}_{01}$-like mode, thanks to its transverse single-lobe field distribution, shown in the inset of Fig. 11a).

Fig. 2 shows the difference $\Delta n_{\text {eff }}$ between the effective index $n_{\text {eff }}$ of the obtained solutions and that of the FM, $n_{\text {eff,FM }}$, as a function of the heat load. With increasing $q$ values, the effective indices of all the modes ramp up quickly, the FM being the one with the highest slope. As a consequence, all the curves in Fig. 2, with the exception of the reference FM one, have a negative slope, which becomes steeper and steeper as much as the mode effective index increases with $q$ slower than the FM. As a reference, the FM $n_{e f f}$ is 1.4499826 with $q=0 \mathrm{~W} / \mathrm{m}$ and 1.4532732 with $q=100 \mathrm{~W} / \mathrm{m}$.

In order to be able to quickly evaluate the confinement degree of each solution, the overlap integral $\Gamma$ over the rare-earth doped region [27] is also shown in Fig. 2] with a color scale. The simultaneous representation of the two quantities allows some insights of the properties of the modes guided into the
TGF. In particular, we can discriminate two different kinds of modes. A first family gathers core-guided modes, which become increasingly confined at higher heat load. In Fig. 2 we can clearly identify, besides the fundamental $\mathrm{LP}_{01}$-like mode, the $\mathrm{LP}_{11}$-like and the $\mathrm{LP}_{21}$-like, whose overlaps reach values higher than 0.4 in the considered $q$ range. Other modes, which are potentially guided in the gain region at higher power levels, are the ones responsible of the brightest purple curves. Notice that the $\Delta n_{\text {eff }}$ curves of the modes belonging to this family are the flattest ones, especially when the $\Gamma$ value is high, meaning that the rate of increase of the effective index with power is proportional to the amount of field in the doped region. A second family of modes which can be clearly identified is the one of the "truly" cladding modes. Their curves are dark, regardless of the heat load, meaning that the overlap over the doped region is low and does not significantly increase with $q$. Moreover, they have the steepest $\Delta n_{\text {eff }}$ curve slope, due to the fact that their field is mostly confined outside from the core.

As already pointed out for PCFs [16], [17], [21], the most confined HOM is mainly determined by the amount of heat load generated in the core. As shown by the electric field distributions 1-4 in Fig. 2 the $\mathrm{LP}_{11}$-like mode is barely guided into the core at low q value, whereas it gets rapidly reconfined as the pump power increases. When the heat load is lower than $24 \mathrm{~W} / \mathrm{m}$ the HOM with the highest $\Gamma$ value is the Bessel-like $L P_{0 n}$ mode shown in inset 5 at $q=10 \mathrm{~W} / \mathrm{m}$, whose high-order resonance on the transverse plane forces a relatively significant amount of field to be kept in the core even when the index gradient across the cross-section is almost negligible.

In order to provide a picture of the modal discrimination which can be achieved with a simple thermally-guiding fiber, the overlap integral over the Yb-doped core of the most relevant modes as a function of $q$ value and pump power is shown in Fig. 3. The grey shaded area of the graph represents the SM regime, which is defined as the region where the FM overlap exceeds 0.8 and the modal discrimination $\Delta \Gamma$ between the $\mathrm{LP}_{01}$-like mode and the $\mathrm{LP}_{11}$-like mode is larger than 0.3 [28]. To the aim of the SM regime definition the other HOMs, which might have a higher $\Gamma$ value over certain heat load range, have been neglected since their excitation or power transfer from the FM is unlikely, due to the effective index mismatch and field distribution, and it was not observed so far. As shown in Fig. 3 the FM is not confined at all in the cold fiber, with $\Gamma<0.3$, which is even lower than the overlap of the $L P_{0 n}$ mode. As the power increases, FM becomes more and more confined, reaching the low- $q$ edge of the SM region for $q=39 \mathrm{~W} / \mathrm{m}$. The $\mathrm{LP}_{11}$-like mode overlap increases with $q$ as well, and the reconfinement of the HOM is faster than the one of the FM for $q>20 \mathrm{~W} / \mathrm{m}$. As a consequence, the modal discrimination decreases, setting the upper limit of the SM region at $q=60 \mathrm{~W} / \mathrm{m}$.

\section{Modal CONTENT OF DC-PCFs UNDER HEAT LOAD}

Before analyzing the effects of the different cladding microstructure on the modal discrimination, it is worth taking a look to the FM effective area $A_{\text {eff }}$ of the considered fibers, 


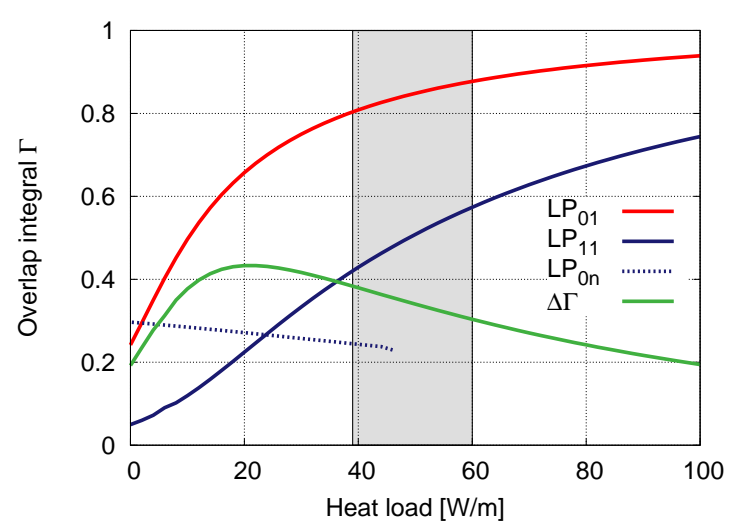

Fig. 3. Overlap integral of the most relevant modes of the TGF.

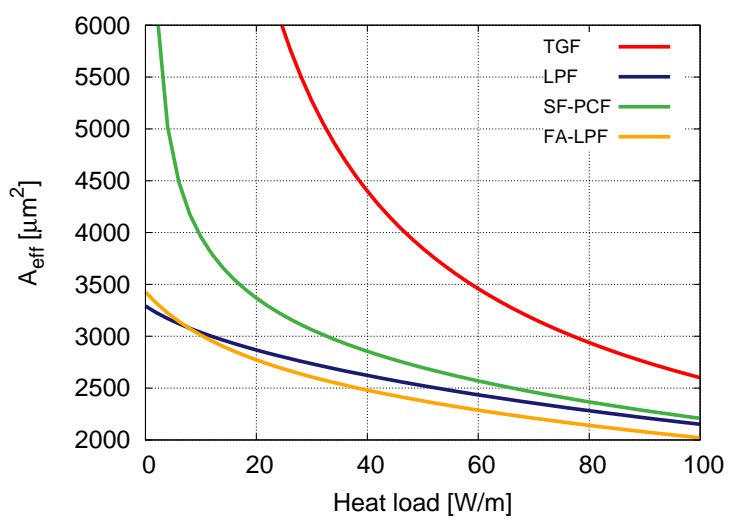

Fig. 4. FM effective area of the considered fibers, as a function of the heat load.

which is shown in Fig. 4 as a function of the heat load. In all cases, thermal lensing has a remarkable impact, which causes the shrinking of the effective area with increasing $q$. As expected, the TGF has the largest effective area, since no other mechanisms but the thermally-induced index gradient contribute to the mode confinement. The microstructured cladding of the considered DC-PCFs provides a further source of mode confinement, which sums to the thermal lensing and turns into an overall lower $A_{\text {eff }}$. At high heat load values, where the contribution of the thermally-induced index gradient on the guidance is stronger, the effective area of all the PCFs is quite similar, allowing a fair comparison between the fibers. On the contrary, at low heat-load values the particular design of the inner cladding governs the mode guidance and effective area. The SF-PCF, due to its smaller cladding holes, allows significant leakage of the FM into the cladding at $q=0 \mathrm{~W} / \mathrm{m}$, which impairs the confinement and causes a quick raise of the $A_{\text {eff }}$ to values above $6000 \mu \mathrm{m}^{2}$. On the contrary, both the LPF and the FA-LPF have similar effective area throughout the graph. Notice that avoided crossings between the FM and cladding modes can occur at low heat load also in the LPF, if slightly different air-clad diameters or wavelengths are considered [29]. In this regard, only the FA-LPF, thanks to its raised-index pedestal, is really insensitive to this detrimental phenomenon.
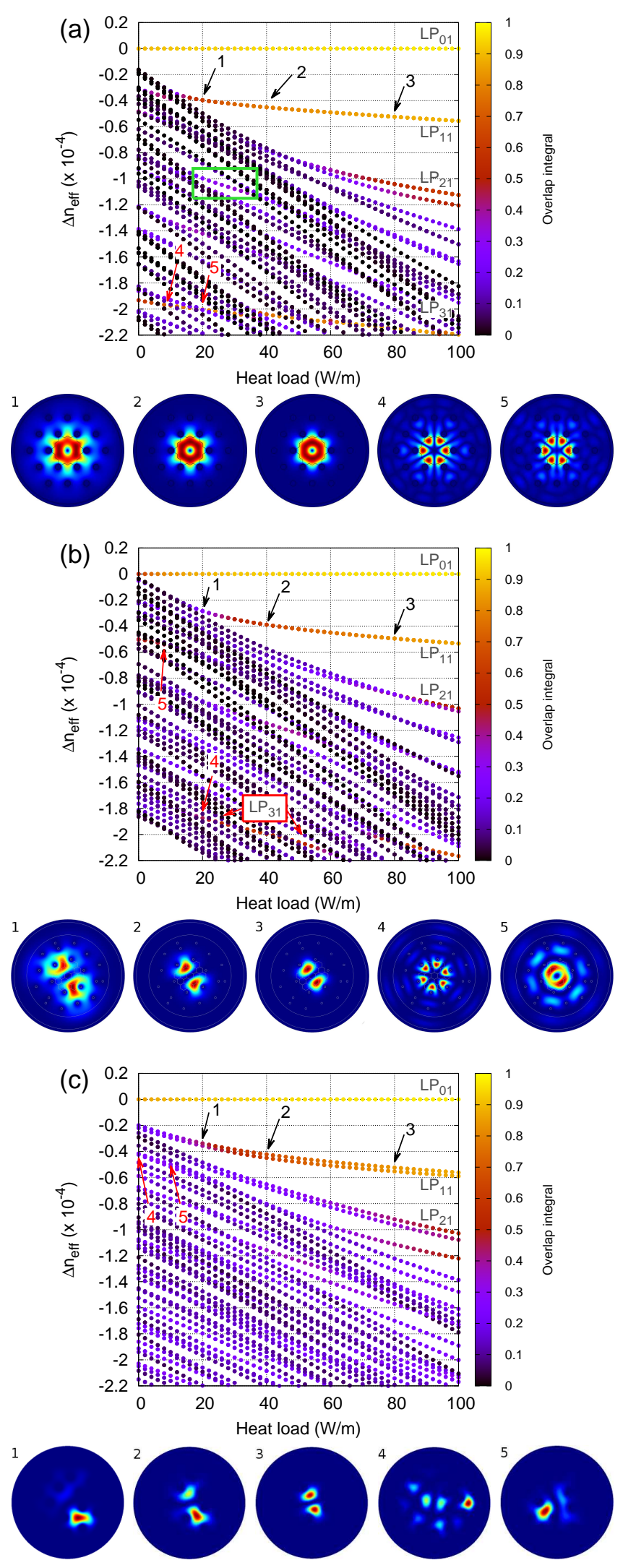

Fig. 5. Map of the modes of (a) LPF, (b) SF-PCF, (c) FA-LPF. Relevant electric field modulus distribution of selected modes within the air cladding are also plotted for each fiber. Videos with animations showing the most relevant mode fields evolution are available for $[\mathrm{LPF}]$ SF-PCF] and $\mathrm{FA}-\mathrm{LPF}$ 

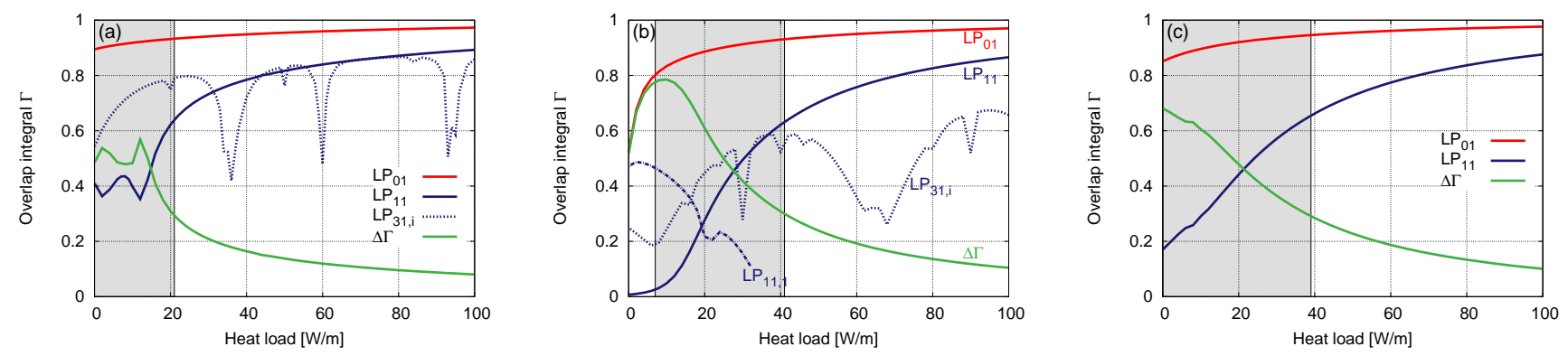

Fig. 6. Overlap integral and modal discrimination of the most relevant modes of the (a) LPF, (b) SF-PCF and (c) FA-LPF, calculated at $\lambda=1032$ nm, as a function of the heat load. Grey shaded area correspond to the SM regime.

Fig. 5]a) shows the $\Delta n_{\text {eff }}$ and, with color scale, overlap integral of the modes with the highest effective index guided into the LPF. From the comparison with Fig. 2 it is possible to infer some comments on the effects of the cladding microstructuration. The most striking effect is on the possibility to clearly discriminate between core-guided and cladding modes, which becomes very difficult to do. Indeed, the $\mathrm{LP}_{01}$ is the only mode that is clearly separated from the others for any value of $q$, and it also has a high overlap even at low power levels. All the other solutions form a sort of cloud where modes with overlap spanning from less than 0.1 to about 0.8 are mixed. Some of them, such as the $\mathrm{LP}_{11}$-like and the $\mathrm{LP}_{21}$-like modes, become increasingly confined with the heat load and, for sufficiently high values of pump power can be clearly distinguished as guided modes. The electric field modulus distribution of the $\mathrm{LP}_{11}$-like mode at $q=20 \mathrm{~W} / \mathrm{m}, 40 \mathrm{~W} / \mathrm{m}$ and $80 \mathrm{~W} / \mathrm{m}$ are shown in subfigures 1,2 and 3 respectively. Other modes have low overlap and steep $\Delta n_{\text {eff }}$ curves regardless of the heat load, similarly to the cladding modes of the TGF. Many of the solutions do not belong to any of the two families that have been identified for the TGF, but are the result of coupling between $\mathrm{LP}_{\nu \mu}$-like core modes and cladding modes. Each core mode couples with many cladding modes, and the specific combination is determined by the value of $q$. When the coupling is weaker, the field distribution of the resulting hybrid mode is more similar to the $\mathrm{LP}_{\nu \mu}$-like mode, yielding to a higher $\Gamma$ value which manifests as "bright spots", such as the one inside the green box in Fig. 5(a). Away from this range, the coupling with the cladding mode is stronger, and avoided crossing-like discontinuities are observed. The most relevant modes belonging to this family are those descending from the hybridization of the $\mathrm{LP}_{31}$-like mode, such as those shown in insets 4 and 5 of Fig. 5(a). Such modes have usually a strong field component in the core and have an overlap comparable with or even higher than the one of the $\mathrm{LP}_{11}$-like mode, especially at low heat load. Notice that, as shown for example in insets 4 and 5, the field distributions of these modes have the same shape in the core and different patterns in the cladding, which depends on the particular cladding mode coupled with the guided mode for that specific heat load value. A clear view of these phenomena can be found in the supporting video LPF. Fig. 5(b) shows the mode map calculated for the SF-PCF. The modes can be parted into the same families already described for LPF, and most of the comments apply as well. The main difference is that, due to the smaller cladding air-filling fraction, all the modes are closer in $n_{e f f}$, which yields to more frequent couplings and worst overall confinement, especially at low power levels. The $\mathrm{LP}_{11}$-like mode, whose electric field modulus distribution is shown in insets 1,2 and 3 for heat load of $20 \mathrm{~W} / \mathrm{m}, 40 \mathrm{~W} / \mathrm{m}$ and $80 \mathrm{~W} / \mathrm{m}$ respectively, can be clearly distinguished from cladding modes only for $q>20 \mathrm{~W} / \mathrm{m}$. Below this threshold the complex interaction between modes causes $\mathrm{LP}_{31}$-cladding and $\mathrm{LP}_{11}$-cladding hybrid modes, shown in insets 4 and 5, to be better confined. Notice that the breaking of the $\mathrm{C}_{6 \nu}$ symmetry and the smaller air-filling fraction are detrimental to the confinement of the modes descending from the $\mathrm{LP}_{31}$-like, which are generally less confined with respect to their counterparts in the LPF. The mode reconfinement and interacion can be clearly observed in the supporting video SF-PCF.

The raised-index pedestal over which the inner cladding of the FA-PCF is built, yields a triple-clad waveguide within which the high-index inner cladding is clearly separated from the air-clad by a pure silica annular region, bringing some remarkable effects on the mode map, shown in Fig. 5(c). Due to the refractive index mismatch, the modes with a relevant fraction of their field in the pure silica ring do not interact with core-guided modes. As a consequence, less avoided crossing-like discontinuities are present in the $\Delta n_{\text {eff }}$ curves. The curve of the "pure" $\mathrm{LP}_{11}$-like HOM, whose field distribution at $q=20 \mathrm{~W} / \mathrm{m}, 40 \mathrm{~W} / \mathrm{m}$ and $80 \mathrm{~W} / \mathrm{m}$ is shown in insets 1-3, can be clearly distinguished from other modes at any heat load, even if its field is poorly confined when $q<15 \mathrm{~W} / \mathrm{m}$. Because of the high degree of asymmetry of this microstructure, symmetric $\mathrm{LP}_{\nu \mu}$ modes do not exist at low heat load, as it can be clearly seen in insets 4 and 5 , which exhibit a certain symmetry as well. As a consequence, these modes are even more weakly confined. Supporting video FA-LPF clearly shows mode reconfinement due to heat load. To wrap up the results shown in Fig. 5, some considerations can be drawn. The first one is that, regardless of the particular design of the inner cladding, three kinds of modes can be observed in DC-PCFs under heat load. The first kind, namely core-guided modes, is characterized by modes with a classic $L P_{\nu \mu}$-like field distribution, which becomes clearer and better confined with increasing heat load. Their overlap integral 
on the core is also monotonically increasing with the pump power. A second kind, namely cladding-modes, groups the solutions with field mainly located in the cladding, which do not significantly penetrate into the core regardless of the heat load. They have very steep $\Delta n_{\text {eff }}$ vs $q$ curves, meaning that their effective index increase with the heat load is much slower than the one of the FM. The last kind, which is not observed in the TGF and it is typical of microstructured fibers, groups hybrid modes, which are generated by coupling of one $L P_{\nu \mu^{-}}$ like core mode with cladding modes. Each core mode couples with several cladding modes and the resulting field distribution is a mix of the two interacting modes with contribution of each determined by the heat load. As a consequence, their overlap on the core increases when the contribution of the $L P_{\nu \mu}$-like core mode becomes predominant, and decreases when coupling with the cladding mode is stronger. In the case where modes involved into these avoided crossings exhibit the minimum effective index difference, they have almost equal overlap. The results shown for three significantly different designs of the inner cladding demonstrate that the choice of the microstructuration has a significant impact on the mode map and particularly on the modes of the latter kind.

A clearer indication on the extent of the SM performances achieved by the considered PCF designs can be inferred from the overlap integral curves, shown in Fig. 6(a)-(c) for the LPF, SF-PCF and FA-LPF respectively. The ranges of $q$ values where each fiber is SM are shaded in grey in the graphs. The FM of the LPF is well confined throughout the whole range of heat load values, with $\Gamma$ steadily above 0.8 . On the contrary, the $\mathrm{LP}_{11}$-like mode is poorly guided at low heat load, and its low overlap grants modal discrimination $\Delta \Gamma>0.3$ up to $q=22 \mathrm{~W} / \mathrm{m}$. Notice that the hybrid modes generated by the coupling of the $\mathrm{LP}_{31}$-like mode with cladding ones, here labeled $\mathrm{LP}_{31, i}$, have an overlap very close to the one of the $\mathrm{LP}_{11}$-like mode throughout most of the considered $q$ range, and they are even better confined for $q<30 \mathrm{~W} / \mathrm{m}$. Minima of the overlap curves are clearly visible for values of $q$ where the coupling with cladding modes is stronger, corresponding to the avoided crossing-like points of Fig. 5(a). Notice that the maximum value of $\Delta \Gamma$ achieved is about 0.55 . Due to the weaker confinement provided by the microstructured cladding of the SF-PCF, the FM is not well guided in the "cold" fiber, with $\Gamma$ only slightly above 0.5 , as shown in Fig. 5(b). The low heat load edge of the SM region is then shifted at $q=8 \mathrm{~W} / \mathrm{m}$, where the thermally-induced parabolic profile provides a sufficient contribution to the FM confinement to reach $\Gamma=0.8$. The $\mathrm{LP}_{11}$-like mode is even more affected than the FM by this effect, and it requires a heat load of about $30 \mathrm{~W} / \mathrm{m}$ to reach an overlap of 0.5 . Indeed, hybrid HOMs, as the one generated from the coupling of cladding modes with the $\mathrm{LP}_{11}$-like one, labeled $\mathrm{LP}_{11,1}$ in Fig. 5(b), and a few generated from the $\mathrm{LP}_{31}$-like mode, labeled $\mathrm{LP}_{31, i}$, are better confined at low power. The $\mathrm{LP}_{11}$-like becomes dominant only with $q>40 \mathrm{~W} / \mathrm{m}$. The upper $q$ limit of the SM region is set at $42 \mathrm{~W} / \mathrm{m}$. Notice that, as already shown elsewhere [30], the SM regime is sensitive to the normalized air-hole diameter. For larger air-holes, it would be shifted towards lower heat load values, becoming more similar to the one of the LPF. As already discussed while analyzing Fig. 5(c), the triple-cladding of the FA-LPF disfavors the coupling between core and cladding modes. As a consequence, hybrid modes do not have a very high overlap integral, which is steadily below the one of $\mathrm{LP}_{11}$-like one. Nevertheless, as shown in Fig. 6(c), the fully-aperiodic cladding provides a remarkable modal discrimination between $\mathrm{LP}_{01}$-like and $\mathrm{LP}_{11}$-like modes, leading to a SM range extending from $0 \mathrm{~W} / \mathrm{m}$ to $38 \mathrm{~W} / \mathrm{m}$, that is to say almost two times wider than for the standard LPF, and maximum $\Delta \Gamma$ up to 0.7 . Notice that in a real fiber amplifier the heat load depends on the pumping scheme and position along the fiber. Equation 1 can be used to provide a rough estimation of the generated heat. Assuming a counterpropagating pump power of $200 \mathrm{~W}$, the corresponding $q$ value will be about $50-60 \mathrm{~W} / \mathrm{m}$ in the last few $\mathrm{cm}$ close to the pumping end, and about $5-10 \mathrm{~W} / \mathrm{m}$ at the opposite fiber end. As a consequence, the considered fibers are effectively SM along most of their length, and slightly multimode at the output end. Notice that the provided results can be used to compare the resilience of the SM regime provided by the different fibers to thermal lensing, but are not easily related to experimentallymeasured TMI thresholds, since the model used does not take into account either propagation of the light inside the amplifier nor the power transfer between modes.

\section{CONCLUSION}

A thorough numerical analysis has been performed to study the effects of thermally-induced refractive index change on the modes which propagate in large mode area double-cladding fibers. A commercial software, based on the finite-element method, has been employed to calculate the temperature and refractive index distribution over the cross-section of three PCFs, which exploit different strategies to enhance modal discrimination and obtain HOM suppression, followed by a modal solver to calculate the guided modes. An analysis of the mode behavior as a function of the heat load has been carried out, which allowed to group the solutions into three different families, with peculiar properties in terms of field distribution and confinement. Furthermore, direct comparison of the SM properties of PCFs with similar FM effective area has been performed, to show the effects of non-symmetric designs on HOM suppression, potentially enabling to push further the threshold for appearance of mode instabilities.

\section{REFERENCES}

[1] D. J. Richardson, J. Nilsson, and W. A. Clarkson, "High power fiber lasers: current status and future perspectives," J. Optics Soc. Am. B, vol. 27, pp. B63-B92, Nov. 2010.

[2] A. Tünnermann, T. Schreiber, and J. Limpert, "Fiber lasers and amplifiers: an ultrafast performance evolution," Applied Optics, vol. 49, pp. F71-F78, Sep. 2010.

[3] M. N. Zervas and C. A. Codemard, "High power fiber lasers: a review," IEEE J. Selected Topics in Quantum Technol., vol. 20, no. 5, p. 0904123, 2014. [Online]. Available: http://ieeexplore.ieee.org/stamp/stamp.jsp?arnumber=6808413

[4] B. Shiner, "The impact of fiber laser technology on the world wide material processing market," in Proc. CLEO 2013, 2013, p. AF2J.1.

[5] C. Zheng, H. Zhang, P. Yan, and M. Gong, "Low repetition rate broadband high energy and peak power nanosecond pulsed Yb-doped fiber amplifier," Optics \& Laser Technology, vol. 49, no. 0, pp. 284-287, 2013. [Online]. Available: http://www.sciencedirect.com/science/article/pii/S0030399213000145 
[6] F. Stutzki, F. Jansen, A. Liem, C. Jauregui, J. Limpert, and A. Tünnermann, "26mJ, $130 \mathrm{~W}$ Q-switched fiber-laser system with near-diffraction-limited beam quality," Opt. Lett., vol. 37, no. 6, pp. 1073-1075, Mar 2012. [Online]. Available: http://ol.osa.org/abstract.cfm?URI=ol-37-6-1073

[7] A. Klenke, S. Hädrich, T. Eidam, J. Rothhardt, M. Kienel, A. Hoffmann, S. Demmler, T. Gottschall, J. Limpert, and A. Tünnermann, "22GW peak power femtosecond fiber CPA system," in Proc. SPIE, vol. 9344, 2015, pp. 93 441A-93441A-6. [Online]. Available: http://dx.doi.org/10.1117/12.2079193

[8] T. Eidam, J. Rothhardt, F. Stutzki, F. Jansen, S. Hädrich, H. Carstens, C. Jauregui, J. Limpert, and A. Tünnermann, "Fiber chirped-pulse amplification system emitting $3.8 \mathrm{GW}$ peak power," Optics Express, vol. 19, no. 1, pp. 255-260, Jan. 2011.

[9] T. T. Alkeskjold, M. Laurila, L. Scolari, and J. Broeng, "Single-mode ytterbium-doped large-mode-area photonic bandgap rod fiber amplifier," Optics Express, vol. 19, no. 8, pp. 7398-7409, Apr. 2011.

[10] A. V. Smith and J. J. Smith, "Mode instability in high power fiber amplifiers," Optics Express, vol. 19, no. 11, pp. 10180-10192, May 2011.

[11] K. R. Hansen, T. T. Alkeskjold, J. Broeng, and J. Lægsgaard, "Thermally induced mode coupling in rare-earth doped fiber amplifiers," Opt. Lett., vol. 37, no. 12, pp. 2382-2384, Jun 2012. [Online]. Available: http://ol.osa.org/abstract.cfm?URI=ol-37-12-2382

[12] L. Dong, "Stimulated thermal rayleigh scattering in optical fibers," Opt. Express, vol. 21, no. 3, pp. 2642-2656, Feb 2013. [Online]. Available: http://www.opticsexpress.org/abstract.cfm?URI=oe-21-3-2642

[13] A. Smith and J. Smith, "Overview of a steady-periodic model of modal instability in fiber amplifiers," Selected Topics in Quantum Electronics, IEEE Journal of, vol. 20, no. 5, pp. 472-483, Sept 2014.

[14] E. Coscelli, F. Poli, T. T. Alkeskjold, M. M. Jørgensen, L. Leick, J. Broeng, A. Cucinotta, and S. Selleri, "Thermal Effects on the SingleMode Regime of Distributed Modal Filtering Rod Fiber," Lightwave Technology, IEEE Journal of, vol. 30, no. 22, pp. 3494-3499, Nov. 2012.

[15] F. Stutzki, F. Jansen, C. Jauregui, J. Limpert, and A. Tünnermann, "Breaking the symmetry for enhanced higher-order mode delocalization," in Proc. SPIE, vol. 8961, 2014, pp. 89611N89 611N-6. [Online]. Available: http://dx.doi.org/10.1117/12.2036901

[16] R. Dauliat, D. Gaponov, A. Benoit, F. Salin, K. Schuster, R. Jamier, and P. Roy, "Inner cladding microstructuration based on symmetry reduction for improvement of singlemode robustness in VLMA fiber," Optics Express, vol. 21, no. 16, pp. 18927-18936, Aug 2013.

[17] E. Coscelli, C. Molardi, M. Masruri, A. Cucinotta, and S. Selleri, "Thermally resilient Tm-doped large mode area photonic crystal fiber with symmetry-free cladding," Optics Express, vol. 22, no. 8, pp. 9707-9714, Apr 2014. [Online]. Available: http://www.opticsexpress.org/abstract.cfm?URI=oe-22-8-9707

[18] F. Stutzki, F. Jansen, T. Eidam, A. Steinmetz, C. Jauregui, J. Limpert, and A. Tünnermann, "High average power large-pitch fiber amplifier with robust single-mode operation," Optics Letters, vol. 36, no. 5, pp. 689-691, Mar. 2011.

[19] A. Benoît, R. Dauliat, R. Jamier, G. Humbert, S. Grimm, K. Schuster, F. Salin, and P. Roy, "Highly efficient higher-order modes filtering into aperiodic very large mode area fibers for single-mode propagation," Opt. Lett., vol. 39, no. 15, pp. 4561-4564, Aug 2014. [Online]. Available: http://ol.osa.org/abstract.cfm?URI=ol-39-15-4561

[20] J. Limpert, T. Schreiber, A. Liem, S. Nolte, H. Zellmer, T. Peschel, V. Guyenot, and A. Tünnermann, "Thermo-optical properties of air-clad photonic crystal fiber lasers in high power operation," Opt. Express, vol. 11, no. 22, pp. 2982-2990, Nov 2003. [Online]. Available: http://www.opticsexpress.org/abstract.cfm?URI=oe-11-22-2982

[21] F. Stutzki, F. Jansen, H.-J. Otto, C. Jauregui, J. Limpert, and A. Tünnermann, "Designing advanced very-large-modearea fibers for power scaling of fiber-laser systems," Optica, vol. 1, no. 4, pp. 233-242, Oct 2014. [Online]. Available: http://www.osapublishing.org/optica/abstract.cfm?URI=optica-1-4-233

[22] F. Poli, E. Coscelli, A. Cucinotta, S. Selleri, and F. Salin, "Single-mode propagation in yb-doped large mode area fibers with reduced cladding symmetry," Photonics Technology Letters, IEEE, vol. 26, no. 24, pp. 2454-2457, Dec 2014.

[23] "Comsol multiphysics 4.3." [Online]. Available: http://www.comsol.com

[24] E. Coscelli and A. Cucinotta, "Modeling thermo-optic effect in large mode area double cladding photonic crystal fibers," International Journal of Modern Physics B, vol. 28, no. 12 , p. 1442002, may 2014. [Online]. Available: http://www.worldscientific.com/doi/abs/10.1142/S0217979214420028
[25] D. C. Brown and H. J. Hoffman, "Thermal, stress, and thermo-optic effects in high average power double-clad silica fiber lasers," Quantum Electronics, IEEE Journal of, vol. 37, no. 2, pp. 207-217, Feb. 2001.

[26] M. M. Johansen, K. R. Hansen, M. Laurila, T. T. Alkeskjold, and J. Lægsgaard, "Estimating modal instability threshold for photonic crystal rod fiber amplifiers," Optics Express, vol. 21, no. 13, pp. 15409 15417, Jul 2013.

[27] A. Cucinotta, F. Poli, S. Selleri, L. Vincetti, and M. Zoboli, "Amplification Properties of $\mathrm{Er}^{3+}$-doped photonic crystal fibers," IEEE/OSA Journal of Lightwave Technology, vol. 21, pp. 782-788, 2003.

[28] M. M. Jørgensen, S. R. Petersen, M. Laurila, J. Lægsgaard, and T. T. Alkeskjold, "Optimizing single mode robustness of the distributed modal filtering rod fiber amplifier," Optics Express, vol. 20, no. 7, pp. 72637273, Mar. 2012.

[29] F. Jansen, F. Stutzki, C. Jauregui, J. Limpert, and A. Tünnermann, "Avoided crossings in photonic crystal fibers," Optics Express, vol. 19, pp. 13 578-13 589, Jul. 2011.

[30] R. Dauliat, E. Coscelli, F. Poli, D. Darwich, A. Benoît, R. Jamier, K. Schuster, S. Grimm, A. Cucinotta, S. Selleri, F. Salin, and P. Roy, "Large mode area aperiodic fiber designs for robust singlemode emission under high thermal load," in Proc. SPIE, vol. 9507, 2015, pp. 9507-1-9507-11. [Online]. Available: http://spie.org/Publications/Proceedings/Paper/10.1117/12.2178935

Enrico Coscelli was born in Reggio Emilia, Italy, in 1982. He received the Laurea Magistrale (M. Sc.) degree (cum laude) in Telecommunication Engineering in 2008 and the Ph.D. in Information Technologies in 2013, both from the University of Parma. His current research activities include photonic crystal fibers, optical fiber sensors, fiber lasers and amplifiers.

Romain Dauliat was born in 1987 in Brive-la-gaillarde, France. He received its $\mathrm{Ph} . \mathrm{D}$. degree in optics from the University of Limoges in 2013 for his work on the development of innovative microstructured optical fibers for high-power application in the Xlim Institute. After spending 15 months as post-doc in the Leibniz Institute for Photonic Technology (Jena, Germany) for the production of these novel fibers and enhancement of glass refractive index control, he is now back to the Xlim institute so as to pursue his research on high power fiber lasers operating either at 1 or $2 \mu \mathrm{m}$.

Federica Poli was born in Reggio Emilia, Italy, in 1977. She received the Laurea degree (five years) cum laude in electronic engineering and the Ph.D. degree in information technology from the University of Parma, Parma, Italy, in 2002 and 2006, respectively. She is currently Assistant Professor at the Department of Information Engineering, University of Parma. Her research interests include photonic crystal fibers, optical fiber amplifiers, optical fiber sensors and integrated optical components.

Dia Darwich was born in Habbouch, Lebanon in 1991; He received the master degree in photonics in 2014 from the University of Lorraine, Metz, France. He is currently $\mathrm{PhD}$ student at the University of Limoges, France. His research activities include $2 \mathrm{~m}$ high power fiber laser as well as the investigation of the thermal load impact in these fiber lasers.

Annamaria Cucinotta was born in Parma, Italy, in 1969. She received the Laurea degree in physics and the Ph.D. degree in information technology from Parma University in 1995 and 1999, respectively. From 2000 to 2003, she was a post-Ph.D. grant holder and a Contract Researcher at the Information Engineering Department, Parma University, where she has lately been Assistant Professor and, since 2014, Associate Professor. Her current research includes optical fiber amplifiers and lasers, photonic-crystal fibers, numerical methods for electromagnetic field analysis.

Stefano Selleri was born in Bologna, Italy, in 1966. He received the Laurea degree in electronic engineering cum laude from Bologna University in 1991 and the Ph.D. degree from Parma University in 1995. Since 1997, he has been a Researcher with the Information Engineering Department, Parma University, where he became Associate Professor in 2002, Full Professor and Head of the Department in 2013. Since 2012, he is Chair of the IEEE Photonics Society Italian Chapter. His research includes numerical methods for electromagnetic field analysis in conventional and photonic-crystal fibers and waveguides, fiber-based amplifiers, optical sensing.

1077-260X (c) 2015 IEEE. Personal use is permitted, but republication/redistribution requires IEEE permission. See http://www.ieee.org/publications_standards/publications/rights/index.html for more information. 
Kay Schuster is focused on design and preparation of special functionalized microstructured fibers for passive, active, and remote-sensing applications. Complex structures of high silica and silicate glass based fibers have been realized for the development of very large mode area laser fibers. In the applicative critical field of special sensor fibers for high temperature applications, he is intensively engaged in the application and alignment of suitable fiber coating materials.

Aurélien Benoît was born in Bordeaux, France, in 1984. He received his $\mathrm{Ph} . \mathrm{D}$. degree in photonics from the University of Limoges, France, in 2015. His current research includes high power amplifiers and lasers based on large mode area fibers and frequency conversion stage in gaz filled hollow-core photonic crystal fibers.

Raphael Jamier received his $\mathrm{PhD}$ degree in 2007 in microwave electronics and optoelectronics (specialty photonics and electronics systems) from the University of Limoges, France. Since 2008, he has been an associate professor at Xlim Research Institute/University of Limoges, where he has been engaged in design, fabrication, and characterization of specialty optical fibers. His current research activities include hollow and all-solid photonic crystal fibers for high-power generation at unconventional spectral domains as ultraviolet or midinfrared wavelengths.

Philippe Roy received his $\mathrm{PhD}$ degree in microwave electronics and optoelectronics (specialty photonics and electronics systems) in 1997 from the University of Limoges. He is now a head of the fiber photonic department at XLIM, which is a mixed laboratory of the University of LIMOGES and CNRS. He is involved in design, fabrication, and characterization of specialty and composite fibers such as air?silica microstructured optical fibers. He develops rare earth?doped fiber with complex structure for high-power fiber laser or nonconventional emitted spectrum.

François Salin has spent 30 years in laser development both at Universities in France and the U.S. and at private companies and has published over 180 papers. He cofounded EOLITE Systems in 2004 to develop and manufacture high-power fiber lasers. He has been the general manager and CTO of EOLITE for 7 years and currently he serves as the director of technology of EOLITE Lasers, part of ESI Inc. 